\title{
3.0 T MRI IVIM-DWI for predicting the efficacy of neoadjuvant chemoradiation for locally advanced rectal cancer
}

\author{
Hongbo Hu ${ }^{1} \cdot$ Huijie Jiang ${ }^{1}$. Song Wang ${ }^{2} \cdot$ Hao Jiang $^{1} \cdot$ Sheng Zhao $^{1} \cdot$ Wenbin Pan ${ }^{1}$
}

Published online: 27 May 2020

(c) The Author(s) 2020

\begin{abstract}
Purpose The purpose of this study was to determine the diagnostic performance of intravoxel incoherent motion (IVIM) on assessing response to neoadjuvant chemoradiation (nCRT) in patients with Locally Advanced Rectal Cancer (LARC). Methods 50 patients with rectal cancer who underwent magnetic resonance (MR) imaging before and after nCRT, the values of pre-nCRT and post-nCRT IVIM-DWI parameters apparent diffusion coefficient (ADC), diffusion coefficient $(D)$, false diffusion coefficient $\left(D^{*}\right)$, and perfusion fraction $(f)$, together with the percentage changes ( $\Delta \%$ parametric value) induced by nCRT were calculated. According to the patient's response to nCRT, the patients were divided into pathological complete response (pCR) and non-pCR groups, Good Response (GR) group and Poor Response (PR) group, and the above values were compared between different groups. Univariate and multiple logistic regression analysis were done to investigate the relation between different parameters and patient nCRT. Draw ROC curve according to sensitivity and specificity, and compare its diagnostic efficacy.

Results There were no significant differences in the baseline data of 50 patients. After nCRT, the ADC and D values for LARC increased significantly (all $p<0.05$ ). The pCR group $(n=9)$ had higher preD*, pref, postD*, $\Delta \%$ ADC and $\Delta \% D$ values than the non-pCR group $(n=41)($ all $p<0.05)$. The GR group $(n=17)$ exhibited higher post $\mathrm{D}, \Delta \% \mathrm{ADC}$ and $\Delta \% D$ values than the PR group $(n=33)$ (all $p<0.05$ ). From the results of Logistic regression analysis found that $\Delta \%$ ADC and $\Delta \% D$ were significantly correlated with patients' response to nCRT. Based on ROC analysis, $\Delta \% D$ had a higher area under the curve value than $\triangle \% \mathrm{ADC}(p=0.009)$ in discriminating the $\mathrm{pCR}$ from non-pCR groups.

Conclusions IVIM-DWI technology may be helpful in identifying the pCR and GR patients to nCRT for LARC.
\end{abstract}

Keywords Rectal cancer · Magnetic resonance imaging · Intravoxel incoherent motion diffusion-weighted imaging · Neoadjuvant chemoradiotherapy

\section{Introduction}

Colorectal cancer (CRC) is one of the most common malignancies with high morbidity and mortality in the world. In recent years, the incidence of colorectal cancer in China has increased year by year, $60-70 \%$ of these cases are of locally advanced rectal cancer (LARC) [1, 2]. LARC includes those cases in which the tumor has spread beyond the wall of the rectum into the surrounding perirectal fat by at least $5 \mathrm{~mm}$

Huijie Jiang

jhjemail@163.com

1 Department of Radiology, The Second Affiliated Hospital of Harbin Medical University, Harbin 150086, China

2 Department of Radiology, Longhua Hospital, Shanghai University of Traditional Chinese Medicine, No. 725, South Wanping Road, Shanghai 200032, China
(T3c-d), when the tumor has invaded local adjacent structures (T4), or when there is involvement of locoregional lymph nodes (N1 or N2) and without distant metastasis (M0), and tumor within $12 \mathrm{~cm}$ from the anal verge $[3,4]$.

Neoadjuvant chemoradiotherapy (nCRT) followed by total mesorectal excision (TME) has become a standard treatment in patients with locally advanced rectal cancer (LARC), which could decrease the loco-regional recurrence rate and even increase overall survival. However, most patients with rectal cancer in China are LARC at the first diagnosis. The current clinical data show that after nCRT and other neoadjuvant therapy, about $20 \%$ of rectal cancer patients can even achieve pathological complete response [5, 6]. However, not all patients can benefit from nCRT, tumor downstaging in patients with LARC, tumor regression differs from patient to patient, ranging from $\mathrm{pCR}$, intermediate regression, or even a complete lack of response. Early detection in poor responders to nCRT could 
provide an opportunity for these patients to proceed directly to surgery, thereby avoiding the morbidity associated with nCRT or to intensive treatment regimens such as second-line chemotherapy or a higher radiation dose to maximize the therapeutic response [7]. Inappropriate treatment will not only delay the operation time, but also increase the risk of distant metastasis.

Imaging studies are frequently used to evaluate patients for screening and staging of colorectal cancer. A recent metaanalysis demonstrated that MRI had inconsistent results in diagnostic performance for restaging rectal cancer after neoadjuvant treatment [8]. Better results were demonstrated when using diffusion-weighted imaging and/or observers with $>5$ years' experience reading rectal/pelvic MRI. DWI can be used to observe the random movement of water molecules in living tissues, and it is widely accepted in the clinic because of its non-invasive nature [9]. DWI and its quantitative parameter ADC, which reflects the spread of rectal cancer, were widely studied by researcher at home and abroad due to their value of predicting and evaluating the curative effect of nCRT for colorectal cancer, but the results are not accepted by everyone. However, in fact, the free motion of water molecules in the human body is affected by many factors and is a non-Gaussian motion, so the ADC value cannot reflect the diffusion of water molecules truly [10]. Some researchers have proposed a more scientific intravoxel incoherent motion model to supplement it [11-13]. The IVIM-DWI is based on the multi-b-valued DWI sequence, and the parameters reflecting microvascular perfusion and diffusion of water molecules in living tissues can be obtained using double exponential model, including true diffusion coefficient $(D)$, false diffusion coefficient $\left(D^{*}\right)$, and perfusion fraction $(f)$. The distinction between true and false perfusion in IVIM-DWI makes up for the deficiency of DWI, reflects the microcirculation perfusion in the capillary network. Therefore, the purpose of this study was to explore the feasibility of IVIM-DWI predicting the effect of nCRT in LARC patients, and analyze whether the distribution of quantitative parameters is different between pCR and non-pCR patients before and after neoadjuvant radiochemotherapy, so as to provide the possibility for follow-up study. Then guide the clinical further screening of colorectal cancer patients suitable for nCRT, to provide more reference for the setting of the individualized treatment plan in the context of multidisciplinary comprehensive treatment, so that patients can benefit more from the treatment.

\section{Materials and methods}

\section{Subject}

This study was reviewed by the ethics committee of our institution, and informed consent was obtained from all patients. This study retrospectively analyzed the data of
50 patients of LARC diagnosed and treated in the hospital from May 2018 to February 2019, including 32 males and 18 females, aged 36 to 63 years, the average age was $(48.4 \pm 15.0)$ years. The inclusion criteria were: (1) Patients with rectal cancer confirmed by colonoscopy biopsy. (2) Patients diagnosed as LARC by imaging examination before treatment. (3) Patients undergoing surgical treatment after nCRT as required in this study. The exclusion criteria were: (1) Patients who received other antineoplastic therapy before nCRT. (2) Incomplete clinical data (3) Patients who fail to complete nCRT treatment. (4) Patients who have not been able to complete the operation.

\section{Experimental equipment and conventional rectal MRI sequence}

All patients underwent conventional MRI examinations and IVIM-DWI. The a GE Discovery MR750w 3.0 T MRI scanner was used to collect the image data by using the phased array body coil, and the conventional sequence and multi-b value DWI sequence were scanned, and the related parameter values were obtained by Function Tool post-processing software analysis. The imaging parameters are summed up in Table 1.

\section{Preparation and scanning methods before the examination}

Before the examination, confirm that the patient is stable and free of contraindications (no metal implants, no claustrophobia, etc.). Explain to the patients before the examination and patients' informed consent. At least half an hour before the examination, the bowel was cleaned without air or any contrast agent. All patients underwent rectal MRI scan and multi- $b$ value DWI examination, no enhanced examination.

\section{Multi-b-valued DWI sequence}

The $b$ values of axial DWI sequence were set at 10 , and 50, 100, 150, 200, 400, 600, 1000, 1500, 2000, $0 \mathrm{~s} / \mathrm{mm}^{2}$.

\section{DWI image post-processing}

Two radiologists with 9 and 12 years of experience in abdominal MRI process the images before and after treatment. Image processing using GE Function Tool postprocessing software after scanning (Fig. 1), the ADC images based on the single exponential model and the pseudo-color images based on the IVIM-DWI quantitative parameters $D, D^{*}$ and $f$ of the double exponential model were selected. Diffusion correlation coefficient $(D)$ and perfusion correlation coefficient $\left(D^{*}, f\right)$ of water 
Table 1 Conventional rectal MRI sequence

\begin{tabular}{|c|c|c|c|c|c|c|}
\hline Parameter & T1WI flair & $\mathrm{T} 2 \mathrm{WI}$ propeller & $\mathrm{T} 2 \mathrm{WI}$ fs propeller & $\mathrm{T} 2 \mathrm{WI}$ propeller & $\mathrm{T} 2 \mathrm{WI}$ propeller & IVIM sequence \\
\hline Acquisition plane & Axial & Axial & Axial & Sagittal & Coronal & Axial \\
\hline Repetition time (ms) & 460 & 5230 & 5400 & 6150 & 4700 & 2000 \\
\hline Echo time (ms) & 23 & 77 & 77 & 72 & 77 & 80 \\
\hline Slice thickness $(\mathrm{mm})$ & 5 & 5 & 5 & 5 & 5 & 5 \\
\hline Slice gap (mm) & 1 & 1 & 1 & 1 & 1 & 1 \\
\hline Field of view $\left(\mathrm{mm}^{2}\right)$ & $250 \times 250$ & $250 \times 250$ & $250 \times 250$ & $270 \times 270$ & $250 \times 250$ & $420 \times 420$ \\
\hline Number of layers & 24 & 24 & 24 & 24 & 18 & 24 \\
\hline Number of excitations & 2 & 2 & 2 & 2 & 2 & 2 \\
\hline Scanning time & $2 \min 20 s$ & $1 \mathrm{~min} 50 \mathrm{~s}$ & $1 \min 35 \mathrm{~s}$ & $2 \min 10 \mathrm{~s}$ & $1 \min 19 \mathrm{~s}$ & $5 \min 48 s$ \\
\hline
\end{tabular}



Fig. 1 Image post processing. Dotted lines show the tumor border

molecules, so as to distinguish the diffusion movement of water molecules in vivo from microcirculation perfusion. The high signal lesion area is selected on the axial DWI image of $b=1000 \mathrm{~s} / \mathrm{mm}^{2}$, and the corresponding level of T2WI image is used as the anatomic structure reference, which requires that the blood vessels, tumor necrosis and bleeding components should not be included, to avoid the influence of heterogeneous components on the measurement results. On the pseudo-color images of each parameter, the solid part of the tumor is selected to outline the region of interest at the maximum level of the tumor and its upper and lower levels (Fig. 2), and then the measurement results of the three-layer region of interest are averaged. The relatively reliable values of ADC $\left(10^{-3} \mathrm{~mm}^{2} / \mathrm{s}\right)$, $D\left(10^{-3} \mathrm{~mm}^{2} / \mathrm{s}\right), D^{*}\left(10^{-3} \mathrm{~mm}^{2} / \mathrm{s}\right)$ and $f$ were obtained. IVIM uses double exponential model and multiple $b$ values

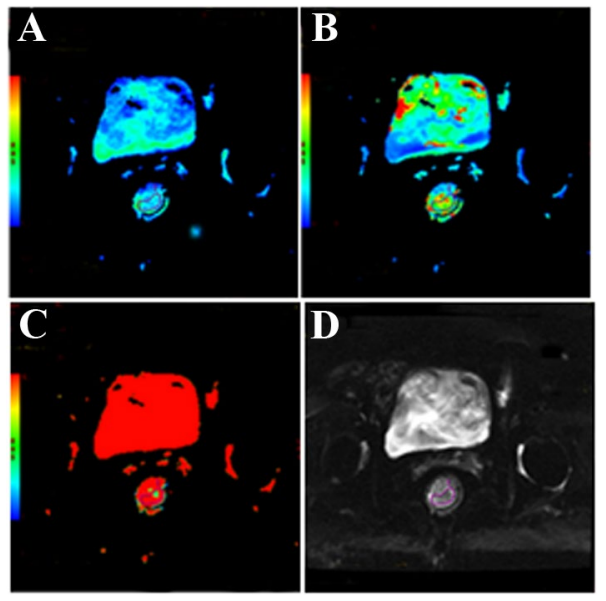

Fig. 2 GE Function Tool post-processing software, image postprocessing: delineate the region of interest, measure the quantitative parameters. a $D$ : pure diffusion coefficient; $\mathbf{b} D^{*}$ : pseudo-diffusion coefficient; $\mathbf{c} f$ : perfusion fraction; d ADC map: apparent diffusion coefficient

to fit and calculate on the basis of DWI, and obtains the diffusion correlation coefficient $(D)$ and perfusion correlation coefficient $\left(D^{*}, f\right)$ of water molecules, so as to distinguish the diffusion movement of water molecules in vivo from microcirculation perfusion. The measured values of the two doctors and the mean value of the two persons were recorded, respectively. In the statistical analysis, the mean value of the two persons with the same observation was taken as the mean value.

\section{Evaluation criteria}

The staging of rectal cancer was determined by referring to the 8th edition of TNM staging criteria published by the International Union against Cancer [14].

Pathological results: the patients without any adenocarcinoma cells in the surgical specimens are $\mathrm{pCR}$, and the rest were non-pCR. The patients were divided into $\mathrm{pCR}$ group 
and non-pCR group according to the postoperative pathological results.

\section{Pathological response evaluation}

TME was performed after post-nCRT MRI examinations. After TME, the fresh specimens were fixed in formalin for $48 \mathrm{~h}$. Tissue sections stained with haematoxylin-eosin were evaluated by two pathologists. Postoperative tumor staging was performed according to the American Joint Committee on Cancer (AJCC) TNM system [15]. The pathologic response induced by nCRT was categorized according to the Dworak tumor regression grade (TRG) system as follows $[16,17]$ : TRG 4 , absence of residual cancer, only a fibrotic mass; TRG 3, presence of rare residual cancer cells scattered through the fibrosis; TRG 2, increased number of residual cancer cells, but still predominating fibrosis; TRG 1, residual cancer outgrowing fibrosis; TRG 0 , absence of regression changes. In this study, the patients with TRG 4 were categorized as the pathological complete responder (pCR) group, whereas the non-pathological responder (non-pCR) group consisted of those with other TRG scores. We also classified the patients into the GR (TRG 3 or 4) and PR (TRG 0, 1 or 2 ) groups.

\section{Univariate and multivariate analysis}

In a univariate analysis, we used the univariate logistic regression analyses to compare the parameters (preADC, preD, preD*, pre $f$, postADC, postD, postD*, post $f, \Delta \% \mathrm{ADC}$, $\Delta \% \mathrm{D}, \Delta \% \mathrm{D} *$ ) between the patients with $\mathrm{pCR}$ group and non-pCR group. If a statistical significance was obtained for more than two parameters among all parameters, these parameters were further analyzed by multivariate logistic regression models to determine whether they had independent predictive value with odds ratios and corresponding 95\% confidence intervals.

\section{ROC analysis}

We used the receiver operating curve (ROC) to evaluate the predictive power of each parameter, and the Youden index (sensitivity, 1-specificity) to determine the sensitivity, specificity and positive predictive value, negative predictive value and diagnosis of each parameter accuracy. $p<0.05$ indicates that the difference is statistically significant.

\section{Statistical analysis}

In this study, EXCEL 2013 was used to establish the database, and SPSS 22.0 statistical software was used to compare the data of the two groups of patients. The measurement data were expressed as $\bar{x} \pm s$. The comparison between the two groups was analyzed by $t$-test, the counting data was expressed by percentage (\%). $p<0.05$ indicated that the difference was statistically significant.

\section{Results}

\section{Baseline data of patients included in the study}

3.1.1. Four patients were excluded from this study due to lack of clinical information $(n=4)$. The cohort of this study eventually included 50 LARC patients. Table 2 lists the clinical characteristics of this cohort.

Table 2 Clinical characteristics of patients in the study

\begin{tabular}{|c|c|}
\hline & Statistics \\
\hline Age (years) & $48.4 \pm 15.0$ \\
\hline \multicolumn{2}{|l|}{ Sex } \\
\hline Male & $32(64.0 \%)$ \\
\hline Female & $18(36.0 \%)$ \\
\hline BMI & $23.8 \pm 3.9$ \\
\hline \multicolumn{2}{|c|}{ Distance of the primary tumor from the anus } \\
\hline $0-5.0 \mathrm{~cm}$ & $13(26.0 \%)$ \\
\hline $5.1-10.0 \mathrm{~cm}$ & $30(60.0 \%)$ \\
\hline $10.1-15.0 \mathrm{~cm}$ & $7(14.0 \%)$ \\
\hline \multicolumn{2}{|l|}{ Hypertension } \\
\hline No & $41(82.0 \%)$ \\
\hline Yes & $9(18.0 \%)$ \\
\hline \multicolumn{2}{|l|}{ Diabetes mellitus } \\
\hline No & $39(78.0 \%)$ \\
\hline Yes & $11(22.0 \%)$ \\
\hline \multicolumn{2}{|l|}{ Smoke } \\
\hline Never smoking & $36(72.0 \%)$ \\
\hline Smoking or smoked previously & $14(28.0 \%)$ \\
\hline \multicolumn{2}{|l|}{ Drinking } \\
\hline Never drinking & $40(80.0 \%)$ \\
\hline Drinking or drank previously & $10(20.0 \%)$ \\
\hline \multicolumn{2}{|c|}{ Post-nCRT pathologic T (ypT) classification } \\
\hline урт0 & 7 \\
\hline ypT1 & 14 \\
\hline урт2 & 12 \\
\hline урТ3 & 9 \\
\hline урт4 & 8 \\
\hline \multicolumn{2}{|l|}{ Pathological response to nCRT } \\
\hline TRG 4 & 9 \\
\hline TRG 3 & 8 \\
\hline TRG 2 & 12 \\
\hline TRG 1 & 13 \\
\hline TRG 0 & 8 \\
\hline
\end{tabular}


Comparison of IVIM-DWI parameters before and after nCRT in different groups

Before nCRT, the patients were examined by MRI, and their IVIM-DWI related values $\left(D, D^{*}\right.$, ADC, $f$ ) were counted. Group patients according to pathological results after neoadjuvant chemotherapy: pCR group and nonpCR group, GR group and PR group. MRI was performed on them. The representative figures of each group are as shown in Fig. 3, 4, 5 and 6. The IVIM related values $\left[D=(1.18 \pm 0.18) \times 10^{-3} \mathrm{~mm}^{2} / \mathrm{s}, D^{*}=(0.89 \pm 0.15) \times 10^{-3}\right.$ $\mathrm{mm}^{2} / \mathrm{s}, \quad$ ADC $=(43.45 \pm 28.63) \times 10^{-3} \mathrm{~mm}^{2} / \mathrm{s}$ and $f=0.23 \pm 0.02]$ were counted, and the differences between before and after treatment $\left[D=(1.75 \pm 0.28) \times 10^{-3}\right.$ $\mathrm{m} \mathrm{m}^{2} / \mathrm{s}, \quad D^{*}=(1.29 \pm 0.47) \times 10^{-3} \mathrm{~mm}^{2} / \mathrm{s}$, $\mathrm{ADC}=(48.57 \pm 33.20) \times 10^{-3} \mathrm{~mm}^{2} / \mathrm{s}$ and $\left.f=0.22 \pm 0.11\right]$ were calculated. Between pre-nCRT and post-nCRT, there were significant differences in the ADC and D values (all $p<0.001$ ), whereas no significant differences were found in the $D^{*}$ and $f$ values ( $p=0.514$ and 0.061 , respectively). It was found that in the pCR and non-pCR groups, as well as GR and PR groups, $\Delta \% \mathrm{D}$ and $\Delta \% \mathrm{ADC}$ value were significant difference before and after treatment $(p<0.05)$. The results are as shown in Fig. 7.
Fig. 3 A patient with LARC from the pCR group. Images in sequence are pre- and post-therapy MR imaging, and IVIMDWI parametric maps. $p C R$ pathological complete response, non- $p C R$ non-pathological complete response; $G R$, good response, $P R$ poor response, $T 2 W I$ T2-weighted imaging, $A D C$ apparent diffusion coefficient, $D$ pure diffusion coefficient, $D^{*}$ pseudo-diffusion coefficient; $f$ : perfusion fraction

Fig. 4 A patient with LARC from the non-pCR group. Images in sequence are preand post-therapy MR imaging, and IVIM-DWI parametric maps. $p C R$ pathological complete response, non- $p C R$ non-pathological complete response; $G R$, good response, $P R$ poor response, $T 2 W I$ T2-weighted imaging, $A D C$ apparent diffusion coefficient, $D$ pure diffusion coefficient, $D^{*}$ pseudo-diffusion coefficient; $f$ : perfusion fraction

Fig. 5 A patient with LARC from the GR group. Images in sequence are pre- and post-therapy MR imaging, and IVIMDWI parametric maps. $p C R$ pathological complete response, non- $p C R$ non-pathological complete response; $G R$, good response, $P R$ poor response, T2WI T2-weighted imaging, $A D C$ apparent diffusion coefficient, $D$ pure diffusion coefficient, $D *$ pseudo-diffusion coefficient; $f$ : perfusion fraction
T2WI

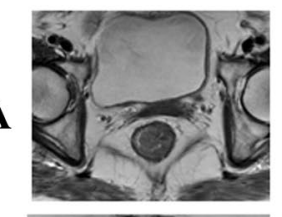

B

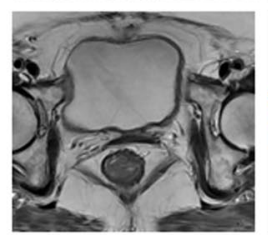

T2WI

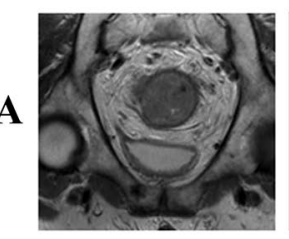

B

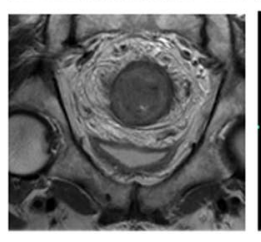

T2WI

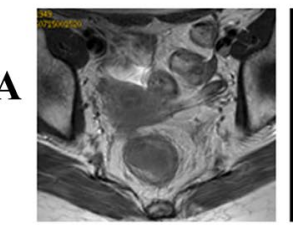

B

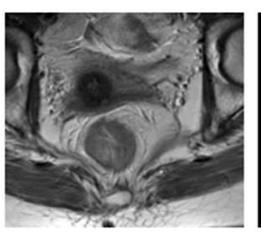

ADC
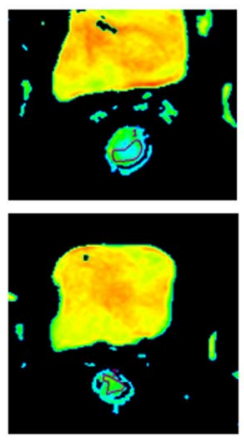

$\mathrm{ADC}$
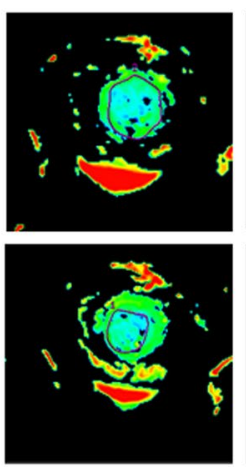

$\mathrm{ADC}$
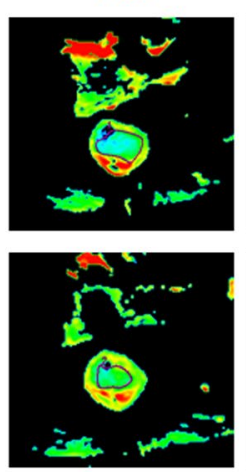

D
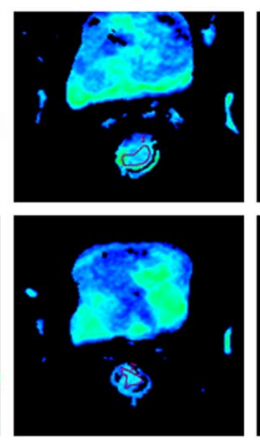

D
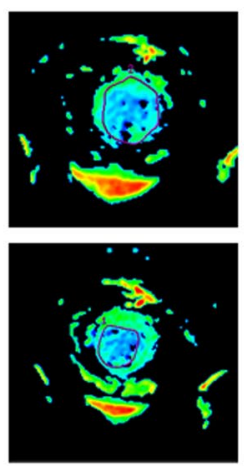

D
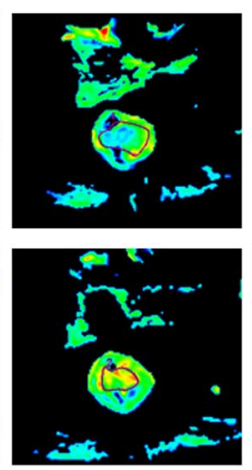

D*
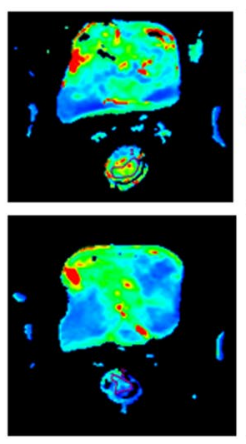

D*
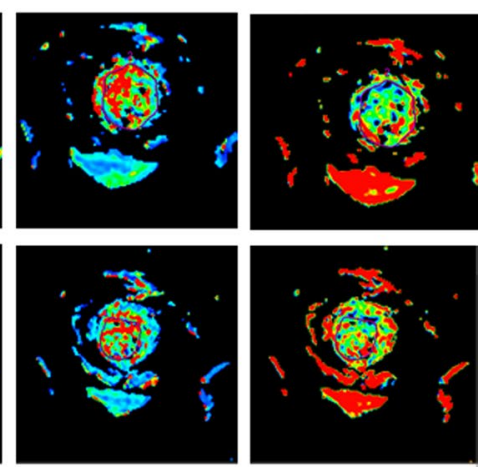

D*
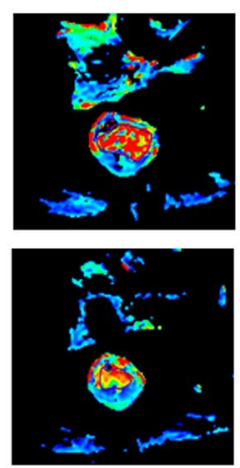

$f$

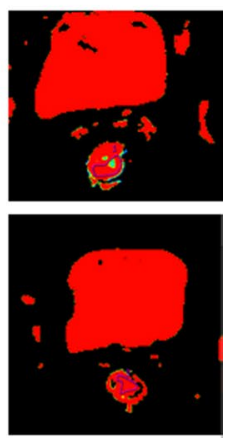

$f$

f
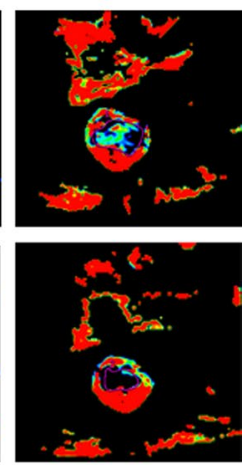
Fig. 6 A patient with LARC from the PR group. Images in sequence are pre- and post-therapy MR imaging, and IVIMDWI parametric maps. $p C R$ pathological complete response, non- $p C R$ non-pathological complete response; $G R$, good response, $P R$ poor response, T2WI T2-weighted imaging, $A D C$ apparent diffusion coefficient, $D$ pure diffusion coefficient, $D^{*}$ pseudo-diffusion coefficient; $f$ perfusion fraction
T2WI

$\mathrm{ADC}$

D

A


$\mathbf{B}$
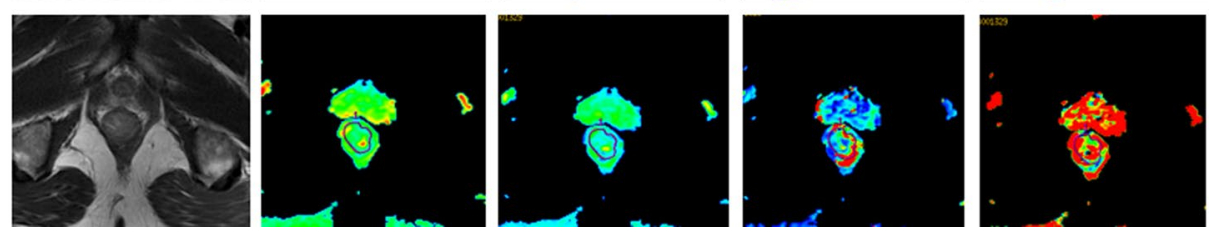

A

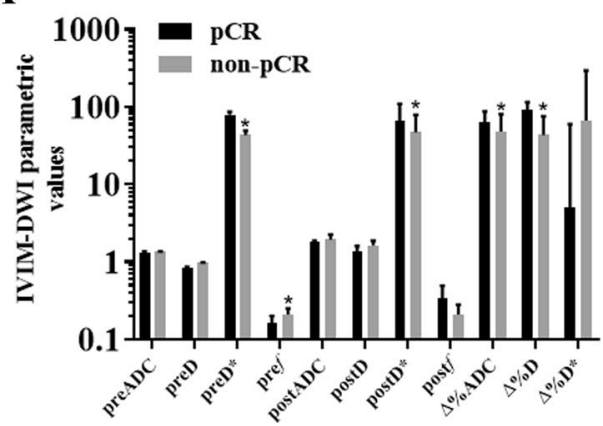

Fig. 7 Differences in the IVIM-DWI parametric values between the pCR and non-pCR groups and between the GR and PR groups. a Difference in the IVIM-DWI parametric values for $\mathrm{pCR}$ and nonpCR; b Difference in the IVIM-DWI parametric values for GR and PR. 1. PreADC; 2. PreD; 3. PreD*; 4. Pref; 5. PostADC; 6. PostD;

\section{Univariate and multivariate logistic regression analysis}

It showed that the preD*, pref, postD*, $\Delta \% \mathrm{D}$ and $\Delta \% \mathrm{ADC}$ values between the $\mathrm{pCR}$ group and the non-pCR group were statistically significant. Multiple logistic regression analysis showed that $\Delta \% D$ value and $\Delta \% \mathrm{ADC}$ value were independent predictors of $\mathrm{pCR}(p=0.034$ and $p=0.009)$, where $\Delta \% D$ value odd ratio was determined 2.42 , the interval of $95 \%$ confidence is $(0.02,10.68) . \Delta \%$ ADC value odd ratio was 5.89 , the interval of $95 \%$ confidence is $(2.56,11.28)$. In contrast, the preD*, pref, and postD* values indicate that it is not an important parameter to predict whether the pathology is completely resolved (all $p>0.05$ ) (Table 3 ).

\section{ROC curve of IVIM-DWI parameters}

Based on ROC curve analysis, the diagnostic performance of the IVIM-DWI parameters in identifying pathological responses are shown in Figs. 8 and 9. To discriminate pCR from non-pCR, $\Delta \% \mathrm{D}$ had the highest area under the curve (AUC) (0. 898), sensitivity and positive predictive value
B

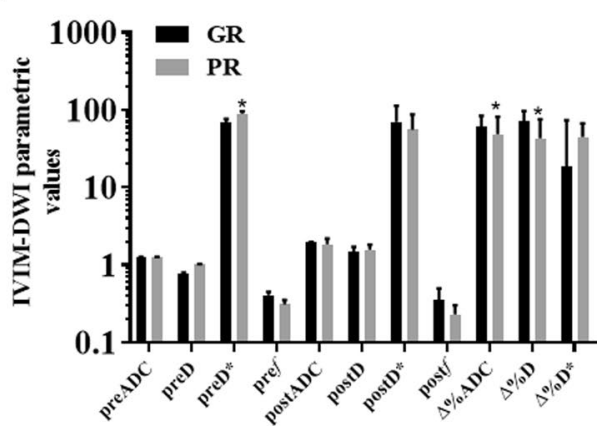

7. PostD*; 8. Postf; 9. $\Delta \% \mathrm{ADC}(\%) ; 10 . \Delta \% \mathrm{D}(\%) ; 11 . \Delta \% \mathrm{D} *(\%) . G R$ good response, $P R$ poor response, $A D C$ apparent diffusion coefficient, $D$ pure diffusion coefficient; $D^{*}$ pseudo-diffusion coefficient, $f$ perfusion fraction

among the five IVIM-DWI parameters (preD*, pref, postD*, $\Delta \% \mathrm{ADC}$ and $\Delta \% D$ ), which could benefit the identification of pCR to nCRT. Among the three IVIM-DWI parameters (postD, $\Delta \% \mathrm{ADC}$ and $\Delta \% D$ ), which were helpful in distinguishing the GR from PR. The postD had the highest specificity and positive predictive value with an AUC of 0.793, whereas $\Delta \% D$ had the highest sensitivity and negative predictive value with an AUC of 0.843 .

\section{Discussion}

MRI is currently an important method for preoperative staging of colorectal cancer [18-20]. It has high accuracy in the location and diagnosis of tumors, judgment of tumor infiltration depth, and determination of resection range. However, the biggest problem based on morphological evaluation is that it can only reflect the intuitive changes of cancer instead of reflect the information of the cell and molecular level accurately. IVIM uses double exponential model and multiple $b$ values to fit and calculate on the 
Table 3 Summarizes the results of univariate and multivariate logistic regression analysis

\begin{tabular}{|c|c|c|}
\hline \multirow[t]{2}{*}{ Parameter } & \multicolumn{2}{|l|}{ Univariate analysis } \\
\hline & Odds ratio & $P$ \\
\hline preADC & $0.17(0.012,18.81)$ & 0.069 \\
\hline preD & $2.46(0.02,10.56)$ & 0.11 \\
\hline preD* & $6.56(0.63,18.13)$ & $0.008 *$ \\
\hline pref & $4.47(0.62,15.1)$ & $0.012 *$ \\
\hline postADC & $0.21(0.08,0.69)$ & 0.510 \\
\hline postD & $3.13(1.24,17.52)$ & 0.066 \\
\hline postD* & $6.12(3.58,21.22)$ & $0.002 *$ \\
\hline post $f$ & $3.66(1.45,16.54)$ & 0.078 \\
\hline$\Delta \% \mathrm{ADC}$ & $5.42(0.52,12.1)$ & $0.023 *$ \\
\hline$\Delta \% D$ & $4.56(4.2,17.25)$ & $0.028 *$ \\
\hline$\Delta \% D^{*}$ & $0.52(0.12,0.51)$ & 0.740 \\
\hline \multirow[t]{2}{*}{ Parameter } & \multicolumn{2}{|l|}{ Multivariate analysis } \\
\hline & Odds ratio & $P$ \\
\hline preD* & $0.74(0.74,1.05)$ & 0.452 \\
\hline pref & $0.05(0.001,24.5)$ & 0.112 \\
\hline postD* & $0.21(0.08,0.69)$ & 0.343 \\
\hline$\Delta \% \mathrm{ADC}$ & $5.89(2.56,11.28)$ & $0.034 *$ \\
\hline$\Delta \% D$ & $2.42(0.02,10.68)$ & $0.009 *$ \\
\hline
\end{tabular}

Correlation between pCR and the IVIM-DWI parametric values

The odds ratio data in parentheses are $95 \%$ confidence intervals. $\mathrm{P}$ value with * mean statistical significance

$p C R$ pathological complete response, IVIM-DWI Intravoxel incoherent motion diffusion-weighted imaging, $A D C$ apparent diffusion coefficient, $D$ pure diffusion coefficient, $D^{*}$ pseudo-diffusion coefficient, $f$ perfusion fraction

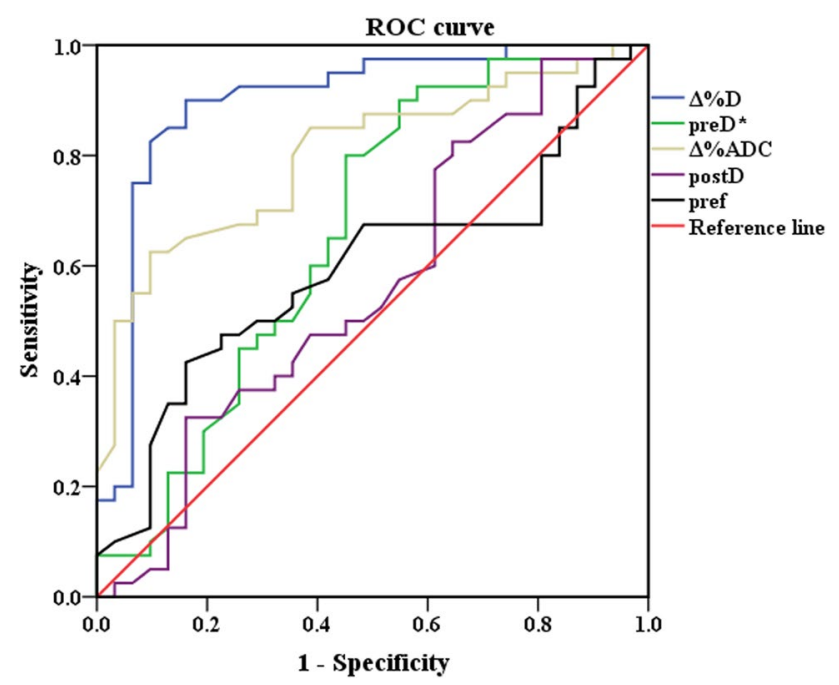

Fig. 8 Receiver operating characteristic curve (ROC) for pCR group and non-pCR group in predicting response to neoadjuvant chemoradiation (nCRT). The area under the curve (AUC) was calculated for ROC curves, and sensitivity and specificity were calculated. The AUC is a measure of accuracy. The closer the curve follows the upper-left border of the ROC space, the more accurate the test. The closer the curve comes to the $45^{\circ}$ diagonal of the ROC space, the less accurate the test

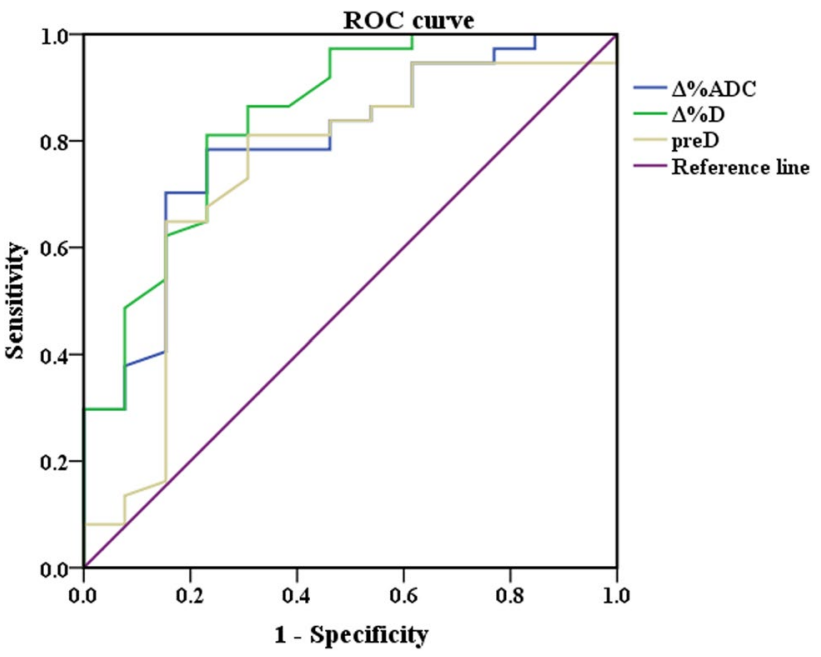

Fig. 9 Receiver operating characteristic curve (ROC) for PR group and GR group in predicting response to neoadjuvant chemoradiation (nCRT). The area under the curve (AUC) was calculated for ROC curves, and sensitivity and specificity were calculated. The AUC is a measure of accuracy. The closer the curve follows the upper-left border of the ROC space, the more accurate the test. The closer the curve comes to the $45^{\circ}$ diagonal of the ROC space, the less accurate the test 
basis of DWI, and obtains the diffusion correlation coefficient $(D)$ and perfusion correlation coefficient $\left(D^{*}, f\right)$ of water molecules, so as to distinguish the diffusion movement of water molecules in vivo from microcirculation perfusion [21-23].

In this study, we focused on the diagnostic efficacy of IVIM-DWI in the evaluation of nCRT in the treatment of LARC, and we evaluated the therapeutic effect of patients through pathological results. In our 50 patient cohort, we found that between pre-nCRT and post-nCRT, there were significant differences in the ADC and D values. The diffusion coefficient (ADC) helps to assess the degree of water molecule diffusion limitation and is related to tumor proliferation, tumor necrosis and other factors [24-26]. Before nCRT, tumor cells proliferated massively, and the cell density increased significantly, which resulted in limited diffusion of water molecules so the ADC value was low [27]. In the macroscopic morphological level, the tumor volume shrinks and the $\mathrm{T}$ stage decreases when the nCRT reaches pCR; in the microscopic molecular level, the tumor cell membrane integrity is destroyed, permeability and necrosis is increased, density and extracellular space is reduced [28].

Our study also found that between the pCR group and the non-pCR group, the difference between preD*, pre $f$, postD*, $\Delta \% D$ and $\Delta \%$ ADC parameters is statistically significant. IVIM-DWI parameters $D^{*}$ and $f$ reflect microvascular perfusion, which can reflect the proportion of false diffusion caused by microcirculation perfusion. The abundance the blood vessels, the greater the value of perfusion-related parameters. The $D^{*}$ and $f$ values of the pCR group before treatment of LARC were higher than those of the non-pCR group, indicating that the lesions in the pCR group had higher microvascular perfusion. Similar studies have found that IVIM-DWI can be used to reflect the histopathological tumor regression grade after nCRT in LARC patients [29], patients with high $f$ value before treatment had good tumor regression performance (specificity 100\%). The value of ADC before treatment could not reflect the curative effect of nCRT.

The $\Delta \% D$ and $\Delta \% \mathrm{ADC}$ value in the pCR group were also higher than that of the non-pCR group, indicating that the cell density of the lesions in the pCR group was smaller and the extracellular space was larger. We also proved that $\Delta \% D$ and $\Delta \% \mathrm{ADC}$ are related to whether the patient's pCR and GR after treatment. They have higher sensitivity and specificity than other values. This discovery will be very meaningful, and it provides a potential independent predictor for clinical IVIM to predict the effect of neoadjuvant chemotherapy on LARC [29-31]. Bates et al. showed that diffusivity derived from the baseline staging MRI, but not diffusion kurtosis or volumetric data, is associated with TRG and therefore shows promise as a potential imaging biomarker to predict the response to neoadjuvant chemotherapy in LARC [32], however in our study we proved that $\Delta \% D$ and $\Delta \%$ ADC related to the TRG, which is a supplement to David's research.

Some limitations of our study need to be carefully considered. (1) The study cohort was small and the results are from a single institution. (2) Respiratory movement and intestinal peristalsis may affect the test results. Although respiratory gating is used, it is inevitable that there will be a slight displacement of organs. (3) Because IVIM-DWI parameters need to be fitted and calculated by DWI data with a wide range of $b$ values, the stability of parameter measurement depends to a large extent on image quality, including signal-to-noise ratio (SNR) and position matching, etc. In the future, we can try to summarize the appropriate scanning methods in order to reduce the impact of scanning factors.

\section{Conclusion}

In conclusion, IVIM technology can predict the efficacy of locally advanced rectal cancer by $\Delta \% D$ and $\Delta \% \mathrm{ADC}$ value. They have a strong correlation with the pathology of patients after nCRT therapy.

Acknwledgements The authors thank all RTs for patient scanning and RNs for patient preparation.

Funding This study was supported by grants from the National Key Research and Development Program of China [2019YFC0118100], National Natural Science Foundation of China [81671760 and 81873910], Scientific Research Transformation Special Fund of Heilongjiang Academy of Medical Sciences [2018415]; Natural Science Foundation of Shanghai[19ZR1457800].

Data Availability The datasets used and/or analysed during the current study are available from the corresponding author on reasonable request.

Code availability Not applicable.

\section{Compliance with ethical standards}

Conflict of interest The authors have no conflicts of interest to disclose.

Ethics approval The study was approved by the ethics committee of the Second Affiliated Hospital of Harbin Medical University (KY2019183).

Consent to participate Written informed consent was obtained prior to the study. 
Consent for publication Written informed consent to publish this information was obtained from study participants.

Open Access This article is licensed under a Creative Commons Attribution 4.0 International License, which permits use, sharing, adaptation, distribution and reproduction in any medium or format, as long as you give appropriate credit to the original author(s) and the source, provide a link to the Creative Commons licence, and indicate if changes were made. The images or other third party material in this article are included in the article's Creative Commons licence, unless indicated otherwise in a credit line to the material. If material is not included in the article's Creative Commons licence and your intended use is not permitted by statutory regulation or exceeds the permitted use, you will need to obtain permission directly from the copyright holder. To view a copy of this licence, visit http://creativecommons.org/licenses/by/4.0/.

\section{References}

1. Pak JN, Jung JH, Park JE, Hwang J, Lee HJ, Shim BS, Kim SH (2020) p53 dependent LGR5 inhibition and caspase 3 activation are critically involved in apoptotic effect of compound $\mathrm{K}$ and its combination therapy potential in HCT116 cells. Phytother Res. https://doi.org/10.1002/ptr.6717

2. MacLeod C, Wilson P, Watson A (2020) Colon capsule endoscopy: an innovative method for detecting colorectal pathology during the Covid-19 pandemic? Colorectal Dis. https://doi. org/10.1111/codi.15134

3. Erika C, De Mattia E, Dreussi E, Montico M, Palazzari E, Navarria F, Bergamo F, Belluco C, Quartuccio L, De Vita S, Canzonieri V, Gagno S, Zanusso C, Buonadonna A, Pucciarelli S, De Paoli A, Toffoli G (2020) Immunogenetic markers in IL17F predict the risk of metastases spread and overall survival in rectal cancer patients treated with neoadjuvant chemoradiotherapy. Radiother Oncol. https://doi.org/10.1016/j.radonc.2020.04.055

4. M DIT, Rosa C, Caravatta L, Augurio A, Borzillo V, S DIS, Perrotti F, Taraborrelli M, Cianci R, Innocenti P, P DIS, Colasante A, Angelucci D, Basti M, Sindici G, Mazzola L, Pizzicannella G, N DIB, Marchioni M, M DIN, Genovesi D (2020) Treatment Intensification for Locally Advanced Rectal Cancer: Impact on Pathological Complete Response and Outcomes. In Vivo 34 (3):1223-1233. https://doi.org/10.21873/invivo.11896

5. Liang HQ, Dong ZY, Liu ZJ, Luo J, Zeng Q, Liao PY, Wu DH (2019) Efficacy and safety of consolidation chemotherapy during the resting period in patients with local advanced rectal cancer. Oncol Lett 17 (2):1655-1663. https://doi.org/10.3892/ ol.2018.9804

6. Lin Z, Liu J, Peng L, Zhang D, Jin M, Wang J, Xue J, Liu H, Zhang T (2018) Complete pathological response following neoadjuvant FOLFOX chemotherapy in BRCA2-mutant locally advanced rectal cancer: a case report. BMC Cancer 18 (1):1253. https://doi.org/10.1186/s12885-018-5182-z

7. Pham TT, Liney GP, Wong K, Barton MB (2017) Functional MRI for quantitative treatment response prediction in locally advanced rectal cancer. Br J Radiol 90 (1072):20151078. https:// doi.org/10.1259/bjr.20151078

8. Maffazzioli L, Zilio MB, Klamt AL, Duarte JA, Mazzini GS, Campos VJ, Chedid MF, Gurski RR (2020) ADC as a predictor of pathologic response to neoadjuvant therapy in esophageal cancer: a systematic review and meta-analysis. Eur Radiol. https ://doi.org/10.1007/s00330-020-06723-x

9. Li YL, Wu LM, Chen XX, Delproposto Z, Hu JN, Xu JR (2014) Is diffusion-weighted MRI superior to FDG-PET or FDG-PET/CT in evaluating and predicting pathological response to preoperative neoadjuvant therapy in patients with rectal cancer? J Dig Dis 15 (10):525-537. https://doi.org/10.1111/1751-2980.12174

10. Dia AA, Hori M, Onishi H, Sakane M, Ota T, Tsuboyama T, Tatsumi M, Okuaki T, Tomiyama N (2017) Application of nonGaussian water diffusional kurtosis imaging in the assessment of uterine tumors: A preliminary study. PLoS One 12 (11):e0188434. https://doi.org/10.1371/journal.pone.0188434

11. Funck C, Laun FB, Wetscherek A (2018) Characterization of the diffusion coefficient of blood. Magn Reson Med 79 (5):27522758. https://doi.org/10.1002/mrm.26919

12. Jerome NP, Miyazaki K, Collins DJ, Orton MR, d'Arcy JA, Wallace T, Moreno L, Pearson AD, Marshall LV, Carceller F, Leach MO, Zacharoulis S, Koh DM (2017) Repeatability of derived parameters from histograms following non-Gaussian diffusion modelling of diffusion-weighted imaging in a paediatric oncological cohort. Eur Radiol 27 (1):345-353. https://doi.org/10.1007/ s00330-016-4318-2

13. Wu WC, Yang SC, Chen YF, Tseng HM, My PC (2017) Simultaneous assessment of cerebral blood volume and diffusion heterogeneity using hybrid IVIM and DK MR imaging: initial experience with brain tumors. Eur Radiol 27 (1):306-314. https://doi. org/10.1007/s00330-016-4272-z

14. Amin MB, Edge SB, Greene FL, et al, eds. AJCC Cancer Staging Manual. 8th ed. New York: Springer; 2017.

15. Li J, Wang J, Pang J, Cao S, Chen J, Xu W (2019) Optimized Parameters of Diffusion-Weighted MRI for Prediction of the Response to Neoadjuvant Chemoradiotherapy for Locally Advanced Rectal Cancer. Biomed Res Int 2019:9392747. https:// doi.org/10.1155/2019/9392747

16. Kang BH, Song C, Kang SB, Lee KW, Lee HS, Kim JS (2020) Nomogram for Predicting the Pathological Tumor Response from Pre-treatment Clinical Characteristics in Rectal Cancer. Anticancer Res 40 (4):2171-2177. https://doi.org/10.21873/anticanres .14177

17. Song C, Chung JH, Kang SB, Kim DW, Oh HK, Lee HS, Kim JW, Lee KW, Kim JH, Kim JS (2018) Impact of Tumor Regression Grade as a Major Prognostic Factor in Locally Advanced Rectal Cancer after Neoadjuvant Chemoradiotherapy: A Proposal for a Modified Staging System. Cancers (Basel) 10 (9). https://doi. org/10.3390/cancers 10090319

18. Meng Y, Zhang C, Zou S, Zhao X, Xu K, Zhang H, Zhou C (2018) MRI texture analysis in predicting treatment response to neoadjuvant chemoradiotherapy in rectal cancer. Oncotarget 9 (15):1199912008. https://doi.org/10.18632/oncotarget.23813

19. Jacobs L, Meek DB, van Heukelom J, Bollen TL, Siersema PD, Smits AB, Tromp E, Los M, Weusten BL, van Lelyveld N (2018) Comparison of MRI and colonoscopy in determining tumor height in rectal cancer. United European Gastroenterol J 6 (1):131-137. https://doi.org/10.1177/2050640617707090

20. Zhang C, Ye F, Liu Y, Ouyang H, Zhao X, Zhang H (2018) Morphologic predictors of pathological complete response to neoadjuvant chemoradiotherapy in locally advanced rectal cancer. Oncotarget 9 (4):4862-4874. https://doi.org/10.18632/oncotarget .23419

21. Kudou M, Nakanishi M, Kuriu Y, Murayama Y, Arita T, Kishimoto M, Konishi E, Goto M, Yamada K, Otsuji E (2020) Value of intra-tumor heterogeneity evaluated by diffusion-weighted MRI for predicting pathological stages and therapeutic responses to chemoradiotherapy in lower rectal cancer. J Cancer 11 (1):168176. doi:https://doi.org/10.7150/jca.38354

22. Peng Y, Li Z, Tang H, Wang Y, Hu X, Shen Y, Hu D (2018) Comparison of reduced field-of-view diffusion-weighted imaging (DWI) and conventional DWI techniques in the assessment of rectal carcinoma at 3.0T: Image quality and histological T staging. 
J Magn Reson Imaging 47 (4):967-975. https://doi.org/10.1002/ jmri.25814

23. Lagemaat MW, Philips BW, Vos EK, van Uden MJ, Futterer JJ, Jenniskens SF, Scheenen TW, Maas MC (2017) Feasibility of Multiparametric Magnetic Resonance Imaging of the Prostate at 7 T. Invest Radiol 52 (5):295-301. https://doi.org/10.1097/ RLI.0000000000000342

24. Bansal S, Gupta NP, Yadav R, Khera R, Ahlawat K, Gautam D, Ahlawat R, Gautam G (2017) Multiparametric magnetic resonance imaging-transrectal ultrasound fusion prostate biopsy: A prospective, single centre study. Indian J Urol 33 (2):134-139. https://doi.org/10.4103/0970-1591.203414

25. Agarwal HK, Mertan FV, Sankineni S, Bernardo M, Senegas J, Keupp J, Daar D, Merino M, Wood BJ, Pinto PA, Choyke PL, Turkbey B (2017) Optimal high b-value for diffusion weighted MRI in diagnosing high risk prostate cancers in the peripheral zone. J Magn Reson Imaging 45 (1):125-131. https://doi. org/10.1002/jmri.25353

26. Hoang JK, Choudhury KR, Chang J, Craciunescu OI, Yoo DS, Brizel DM (2014) Diffusion-weighted imaging for head and neck squamous cell carcinoma: quantifying repeatability to understand early treatment-induced change. AJR Am J Roentgenol 203 (5):1104-1108. https://doi.org/10.2214/AJR.14.12838

27. Chandramohan A, Siddiqi UM, Mittal R, Eapen A, Jesudason MR, Ram TS, Singh A, Masih D (2020) Diffusion weighted imaging improves diagnostic ability of MRI for determining complete response to neoadjuvant therapy in locally advanced rectal cancer. Eur J Radiol Open 7:100223. https://doi.org/10.1016/j. ejro.2020.100223

28. Capelli G, De Simone I, Spolverato G, Cinquini M, Moschetti I, Lonardi S, Masi G, Carlomagno C, Corsi D, Luppi G, Gambacorta MA, Valvo F, Cannizzaro R, Grillo F, Barbaro B, Restivo A, Messina M, Pastorino A, Aschele C, Pucciarelli S (2020) Non-Operative Management Versus Total Mesorectal Excision for Locally Advanced Rectal Cancer with Clinical Complete Response After Neoadjuvant Chemoradiotherapy: a GRADE Approach by the Rectal Cancer Guidelines Writing Group of the Italian Association of Medical Oncology (AIOM). J Gastrointest Surg. https://doi.org/10.1007/s11605-020-04635-1

29. Bakke KM, Hole KH, Dueland S, Groholt KK, Flatmark K, Ree AH, Seierstad T, Redalen KR (2017) Diffusion-weighted magnetic resonance imaging of rectal cancer: tumour volume and perfusion fraction predict chemoradiotherapy response and survival. Acta Oncol 56 (6):813-818. https://doi.org/10.1080/02841 86X.2017.1287951

30. Liang CY, Chen MD, Zhao XX, Yan CG, Mei YJ, Xu YK (2019) Multiple mathematical models of diffusion-weighted magnetic resonance imaging combined with prognostic factors for assessing the response to neoadjuvant chemotherapy and radiation therapy in locally advanced rectal cancer. Eur J Radiol 110:249-255. https ://doi.org/10.1016/j.ejrad.2018.12.005

31. Xu Q, Xu Y, Sun H, Chan Q, Shi K, Song A, Wang W (2018) Quantitative intravoxel incoherent motion parameters derived from whole-tumor volume for assessing pathological complete response to neoadjuvant chemotherapy in locally advanced rectal cancer. J Magn Reson Imaging 48 (1):248-258. https://doi. org/10.1002/jmri.25931

32. Bates DDB, Mazaheri Y, Lobaugh S, Golia Pernicka JS, Paroder V, Shia J, Zheng J, Capanu M, Petkovska I, Gollub MJ (2019) Evaluation of diffusion kurtosis and diffusivity from baseline staging MRI as predictive biomarkers for response to neoadjuvant chemoradiation in locally advanced rectal cancer. Abdom Radiol (NY) 44 (11):3701-3708. https://doi.org/10.1007/s00261-01902073-5

Publisher's Note Springer Nature remains neutral with regard to jurisdictional claims in published maps and institutional affiliations. 